\title{
Guaiane 的骨架结构合成研究
}

\author{
王敏寿*＼cjkstart张洪涁* \\ ( ${ }^{a}$ 滇西应用技术大学理化教研室 云南大理 671006) \\ ( ${ }^{b}$ 云南大学自然资源药物化学教育部重点实验室 昆明 650091)
}

\begin{abstract}
摘要 报道了一种基于 Prins 环化反应合成手性五元并七元氧桥环结构的方法, 为愈创木烷型倍半萜类天然产物骨架 结构的合成提供了一种新思路. 该合成工作是以 dioxinone 为基础, 利用路易斯酸诱导发生分子内的 Prins 环化反应，以 两步 72\%的联合收率实现了手性[5,7]并环氧桥环的合成.

关键词 愈创木烷; 手性氧桥七元环; Prins 反应
\end{abstract}

\section{Synthesis of Framework Structure of Guaiane}

\author{
Wang, Minshou* Zhang, Hongbin*
}

( ${ }^{a}$ West Yunnan University of Applied Sciences, Department of Physics and Chemistry, Dali, Yunnan 671006)

$\left({ }^{b}\right.$ Key Laboratory of Medicinal Chemistry for Natural Resource, Ministry of Education, Yunnan University, Kunming 650091)

\begin{abstract}
A new method for the synthesis of chiral 5,7-fused oxygen bridged ring based on Prins cyclization is reported. It provides a new way for the synthesis of the skeleton structure of guaiacane sesquiterpenes. Based on dioxinone, the synthesis of chiral 5,7-fused oxygen bridged ring is achieved in a two-step combined yield of $72 \%$ by using Lewis acid induced intramolecular Prins cyclization.

Keywords guaiane; chiral oxygen bridge seven-membered ring; Prins reaction
\end{abstract}

愈创木烷(Guaiane)型倍半萜类天然产物具有典型 的 [5,7]并环结构及多样的生物活性，在自然界中占有举 足轻重的地位. 目前, 已经分离并鉴定了结构的愈创木 烷型倍半萜类化合物已逾数百种 ${ }^{[1]}$.

从大戟科植物 Phyllanthus engleri 的根皮和叶皮中 分离得到的 Englerin A (1), 展示出了强大的肾癌细胞抑 制活性 ${ }^{[2]}$. 从 Decachaeta scabrella 植物的叶子中分离的 得到的 Achalensolide (2)具有抑制人体胎盘微粒体的芳 香酶活性 ${ }^{[3]}$. 从薄荷科植物 Pogostemon colelin 的天竺薄 荷油中分离得到的 Pogostol (4), 具有良好的呕吐抑制活 性 ${ }^{[4]}$. 愈创木烷型倍半萜类天然产物所表现出的多方面 重要生物活性及其独特的骨架结构 ${ }^{[5]}$, 吸引了众多有机 合成工作者的研究兴趣.

迄今为止, 已经开发出来了多种方法学用于 [5,7]并 环结构单元的愈创木烷型倍半萜类天然产物的合成研 究. 例如: 铑催化的 1,3 偶极环加成反应 ${ }^{[6]}$ (Scheme 1, a)、铑催化的 Pauson-Khand 反应 ${ }^{[7] 、}$ 、烯烃复分解反应 ${ }^{[8]}$ 、 路易斯酸诱导的 Michael/coniaene 反应 ${ }^{[0]}$ 、铂催化的 [4
$+3]^{1}$ 环反应 ${ }^{[10]} 、 \mathrm{SmI}_{2}$ 诱导的单电子还原内酯环化反应 ${ }^{[11]}$ 以及分子内羟醛缩合环化反应 ${ }^{[12]}$.

上述构建 $[5,7]$ 并环结构的方法中, 大多数都需要用 到贵金属催化剂, 例如: 铑、钓和钐等金属的催化剂. 由 于贵金属催化剂的使用，导致合成成本的大幅度提高.

2018 年, 李志成研究组 ${ }^{[13]}$ 发展了一种快速构建 [5,7]并环结构的方法(Scheme 1, b). 该方法是通过路易 斯酸诱导 Chan 式二烯体与双羰基化合物发生了 Aldol 缩合串联 Prins 环化反应, 实现了 $[5,7]$ 并环结构的高效 合成.

由于 Chan 式二烯体的易挥发性、不稳定性和长时 间储存易变质性，使其在合成中的应用受到了很大的限 制. 为了降低合成成本(尽量少用贵金属催化剂), 同时 解决 Chan 式二烯体难操作和易变质的问题, 本研究组 通过研究发现将 dioxinone (2,2,6-trimethyl-4H-1,3-dioxin-4-one)化合物应用 Prins 环化反应，可以妥善地解决 上述问题.

\footnotetext{
* Corresponding authors. E-mail: wmshou.good@163.com; zhanghb@ynu.edu.cn

Received September 4, 2020; revised November 9, 2020; published online December 3, 2020.
} 
<smiles>CC(C)[C@@]12C[C@H](OC(=O)CO)[C@@](C)(O1)[C@H]1CC[C@@H](C)[C@H]1[C@H]2OC(=O)/C=C/c1ccccc1</smiles>

Englerin A (1)<smiles>C=C(CO)C(=O)O[C@H]1CC(=C)[C@H]2C[C@H]3OC(=O)O[C@@]3(CO)C2[C@H]2CC(=O)C(=C)[C@@H]12</smiles>

Hololeucin (3)

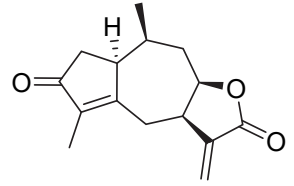

Achalensolide (2)

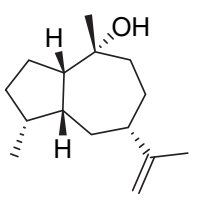

Pogostol (4)
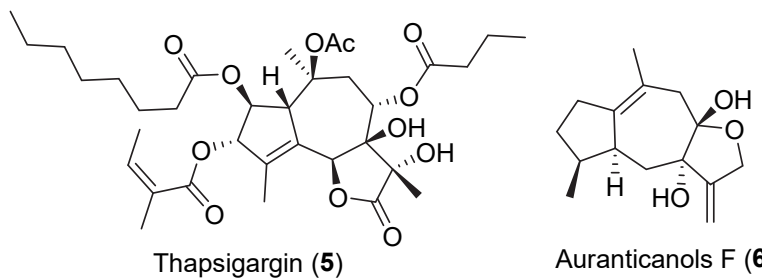

Auranticanols F (6)

图 1 代表性愈创木烷型倍半萜类天然产物

Figure 1 Representative guaianes-type sesquiterpenoid natural products

(a) Previous work: 1,3-dipolar cycloaddition

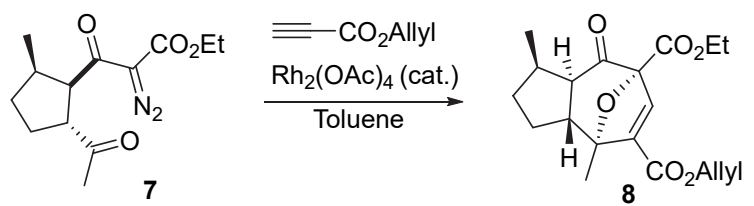

(b) Lee's work: Aldol-addition-Prins-cyclization approach

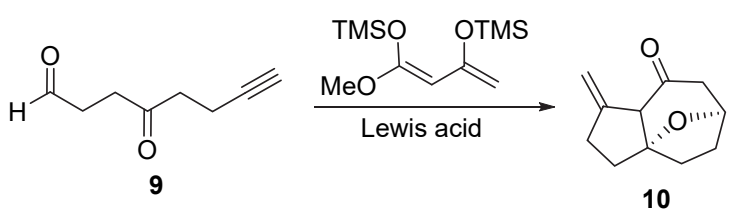

(c) This work: practical construction of 5,7-fused ring

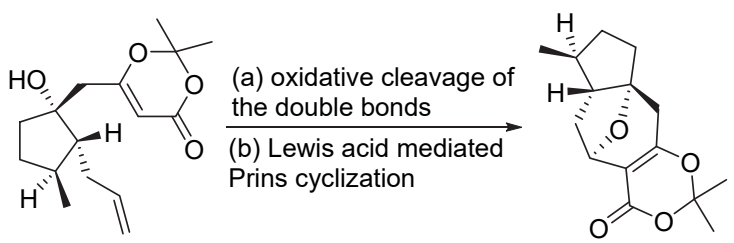

图式 1 合成 5,7 并环的代表性例子和本研究组的工作

Scheme 1 Representative examples of synthesizing 5,7-fused ring and our work

本研究组一直进行 dioxinone 化合物的研究及其在 合成中的应用 ${ }^{[14]}$. Dioxinone 化合物在常温下能够稳定 存在, 且具有一定的耐酸性, 方便进行结构修饰, 酯化 和酰胺化反应都能够得到较好的收率, 操作和应用起来 十分方便.

\section{1 结果与讨论}

该合成是在之前工作的基础上进行的. 首先从商业 易得的 2-氧代环戊羧酸乙酯(11)出发, 参照文献[15]引 入烯丙基得到化合物 12, 紧接着在本研究组 ${ }^{[16]}$ 发展的 条件下，与 dioxinone 发生亲核加成反应，以 $80 \%$ 的收率 得到化合物 13. 将化合物 13 经臭氧化反应和路易斯诱 导的 Prins 环化反应后, 成功地实现了五元并七元氧桥 环的构建, 得到了化合物 14 (Scheme 2). 化合物 14 经过 单晶 $\mathrm{X}$ 射线的测试，证实了其 [5,7]并环结构及其立体化 学关系 ${ }^{[17]}$. 该化合物的主体环系结构由五元环并七元 氧桥环组成, 其中五元环和七元环结构呈反式稠合关 系，乙氧酰基与氧桥呈反式关系.
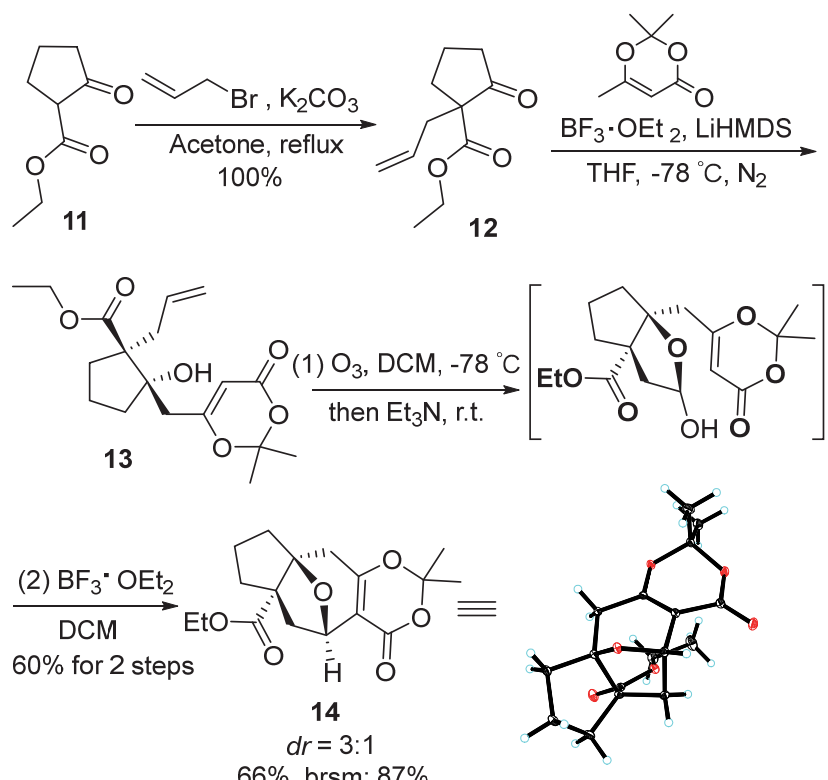

图式 2 目标化合物 14 的合成路线

Scheme 2 Synthetic route of target compound 14

在证实了利用 dioxinone 化合物经历分子内的 Prins 环化反应可以构建五元并七元氧桥环化合物后，作者开 始检验该方法在克制级合成不对称氧桥环化合物的应 用效果. 参照文献[18]将长叶薄荷酮经过两步结构改造 后的 $\beta$ 酮酸酯化合物 15 引入烯丙基，得到化合物 16. 化 合物 16 在表 1 中所列的条件下与 dioxinone 进行反应尝 试，都不能得到预期的化合物 17. 分析原因认为, 预期 的化合物 17 自身的空间位阻太大，难以合成，于是，将 化合物 16 的酯基进行脱除. 化合物 16 在 Krapcho 脱 羧 ${ }^{[19]}$ 条件下得到酮羰基化合物 18. 酮羰基化合物 18 在 $\mathrm{BF}_{3} \cdot \mathrm{OEt}_{2}$ 和 LiHMDS 双活化条件下，与 dioxinone 发生 亲核加成反应得到羟基化合物 19. 化合物 19 经历臭氧 化反应以及 $\mathrm{BF}_{3} \cdot \mathrm{OEt}_{2}$ 诱导的分子内 Prins 环化反应，以 $72 \%$ 的收率实现手性五元并七元氧桥环化合物 20 的合 成(Scheme 3). 
表 1 化合物 17 的合成尝试 $a$

Table 1 Attempt to synthesize compound $\mathbf{1 7}$<smiles>CCOC(=O)C1C(=O)CC[C@H]1C</smiles>

15

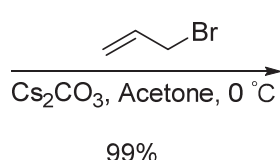

$99 \%$

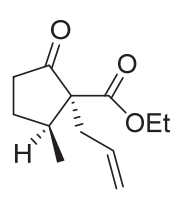

16

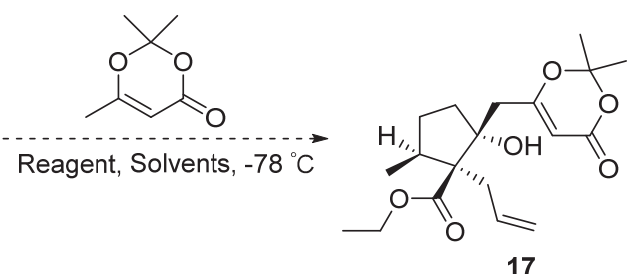

17

\begin{tabular}{|c|c|c|c|c|}
\hline Entry & Condition $^{b, c}$ & Substrate $^{d}$ & Tempreature $/{ }^{\circ} \mathrm{C}$ & Result \\
\hline 1 & LiHMDS, $\mathrm{ZnCl}_{2}, \mathrm{~N}_{2}$ & Dioxinone & -78 & No reaction \\
\hline 2 & KHMDS, $\mathrm{ZnCl}_{2}, \mathrm{~N}_{2}$ & Dioxinone & -78 & No reaction \\
\hline 3 & $\mathrm{NaHMDS} \mathrm{BF}_{3} \cdot \mathrm{OEt}_{2}, \mathrm{~N}_{2}$ & Dioxinone & -78 & No reaction \\
\hline 4 & $\mathrm{LiHMDS} \mathrm{BF}_{3} \bullet \mathrm{OEt}_{2}, \mathrm{~N}_{2}$ & Dioxinone & -78 & No reaction \\
\hline 5 & $\mathrm{KHMDS}, \mathrm{BF}_{3} \cdot \mathrm{OEt}_{2}, \mathrm{~N}_{2}$ & Dioxinone & -78 & No reaction \\
\hline
\end{tabular}

${ }^{a}$ General procedures were followed. ${ }^{b} 2.0$ equiv. of base (LiHMDS, NaHMDS or KHMDS) was used. ${ }^{c} 2.0$ equiv. of Lewis acid $\left(\mathrm{ZnCl}_{2}\right.$ or BF $\left._{3} \cdot \mathrm{OEt}_{2}\right)$ was used. ${ }^{d} 1.5$ equiv. of dioxinone was used.<smiles>C=CCC1(C(=O)OCC)C(=O)CC[C@H]1C</smiles>

16

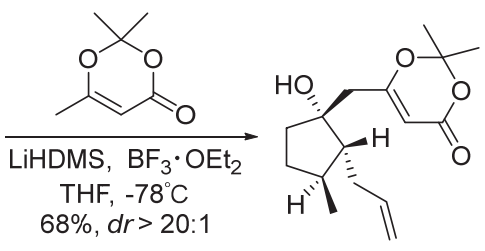

19

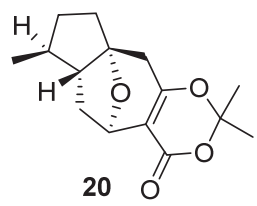

图式 3 目标化合物 20 的合成路线

Scheme 3 Synthetic route of target compound 20

化合物 20 的成功合成, 实现了手性五元并七元氧 桥环的构建, 该环的主体环系与愈创木烷型倍半萜类天 然产物的 $[5,7]$ 并环结构相似. 该研究结果也为本研究组 后续进行愈创木烷型倍半萜类天然产物的合成奠定了 基础.

\section{2 结论}

愈创木烷型倍半萜类天然产物具有多方面的生物 活性和潜在的药用价值, 开发多种高效的合成方法, 对 该类天然产物的合成研究意义非凡. 本课题组发展了一 种实用且直接的合成手性五元并七元氧桥环的方法, 为 深入研究愈创木烷型萜类天然产物的普适性合成奠定 了基础.

\section{3 实验部分}

\section{1 仪器与试剂}

Avance 300、400M 核磁共振仪(以 $\mathrm{TMS}$ 为内标, $\mathrm{CDCl}_{3}$ 为溶剂, 德国 Bruker 公司); $\mathrm{AB}-\mathrm{QSTAR}$ 脉冲星质 谱仪或 Agilentg6230 飞行时间质谱仪; UV-210A 光谱仪 测量旋光度; RY-1 型熔点仪(天津分析仪器厂). 反应 中使用的甲苯和四氢呋喃均是经过金属钠干燥后蒸 馏所得; 二氯甲烷由氢化钙或 $\mathrm{P}_{2} \mathrm{O}_{5}$ 蒸馏所得. 反应 中使用的起始材料和试剂均从 Acros、Aldrich、 Adamas-beta ${ }^{2}$ 等试剂公司购买所得，除非另有说明， 否则未经纯化即可使用.

\section{2 实验方法}

\section{2 .1 化合物 14 的合成}

参照文献[15]合成中间体化合物 12. 将化合物 12 (348.5 mg, $2.00 \mathrm{mmol}$ )加入干燥的 $50 \mathrm{~mL}$ 双口瓶中, 在 氮气保护下, 往瓶中加入干燥的四氢呋喃(THF) (10 $\mathrm{mL})$. 在 $-78{ }^{\circ} \mathrm{C}$ 条件下, 向反应液中用注射器加入无水 的 $\mathrm{BF}_{3} \cdot \mathrm{OEt}_{2}(253 \mu \mathrm{L}, 2.00 \mathrm{mmol})$, 搅拌 $1 \mathrm{~h}$. 与此同时, 将 dioxinone (369.6 mg, $2.60 \mathrm{mmol}$ )加入另外一个干燥的 $50 \mathrm{~mL}$ 双口瓶中, 在氮气保护下, 往瓶中加入干燥的 THF $(10 \mathrm{~mL})$. 在 $-78{ }^{\circ} \mathrm{C}$ 条件下, 向反应液中用注射器 加入 LiHMDS (1.0 mol/L in THF, $2.60 \mathrm{~mL}, 2.60 \mathrm{mmol})$. 反应 $1 \mathrm{~h}$ 后, 在 $-78{ }^{\circ} \mathrm{C}$ 条件下, 用双头针的两头分别插 入两个双口瓶的胶塞中，抽气，使 $<2>$ 中的反应液导 流到 $<1>$ 中, 维持在 $-78{ }^{\circ} \mathrm{C}$ 继续反应 $3 \mathrm{~h}$. 滴加饱和 氯化铵 $(10 \mathrm{~mL})$ 淬灭反应, 加入水 $(10 \mathrm{~mL})$ 和乙酸乙酯 $(20$ $\mathrm{mL})$ 进行萃取. 水相继续用乙酸乙酯 $(10 \mathrm{~mL} \times 2)$ 萃取, 合并有机相, 用无水硫酸镁干燥, 过滤, 用旋转蒸发仪 蒸干溶剂, 硅胶柱层析 $[V$ (石油醚 $): V($ 乙酸乙酯 $)=3$ : 
1], 分离得到 $148.7 \mathrm{mg}$ 1-烯丙基-2-((2,2-二甲基-4-羰基4H-1,3-二氧环己烯基)甲基)-2-羟基环戌烷甲酸乙酯 (13): 无色油状液体, 收率 65\%, brsm: $87 \%, d r=3: 1$. ${ }^{1} \mathrm{H}$ NMR (400 MHz, $\left.\mathrm{CDCl}_{3}\right) \delta: 5.66 \sim 5.55(\mathrm{~m}, 1 \mathrm{H}), 5.28$ $(\mathrm{s}, 1 \mathrm{H}), 5.10 \sim 4.93(\mathrm{~m}, 2 \mathrm{H}), 4.20 \sim 4.02(\mathrm{~m}, 2 \mathrm{H}), 2.67(\mathrm{dd}$, $J=13.7,7.0 \mathrm{~Hz}, 1 \mathrm{H}), 2.56$ (s, 1H), 2.41 (d, $J=14.1 \mathrm{~Hz}$, $1 \mathrm{H}), 2.25$ (d, $J=14.1 \mathrm{~Hz}, 1 \mathrm{H}), 2.20$ (dd, $J=13.6,7.6 \mathrm{~Hz}$, $1 \mathrm{H}), 2.14 \sim 2.01(\mathrm{~m}, 1 \mathrm{H}), 1.93 \sim 1.85(\mathrm{~m}, 1 \mathrm{H}), 1.81 \sim 1.67$ (m, 4H), $1.64(\mathrm{~s}, 3 \mathrm{H}), 1.64(\mathrm{~s}, 3 \mathrm{H}), 1.22(\mathrm{t}, J=7.1 \mathrm{~Hz}, 3 \mathrm{H})$; ${ }^{13} \mathrm{C}$ NMR $\left(100 \mathrm{MHz}, \mathrm{CDCl}_{3}\right) \delta: 174.84,168.97,161.11$, 134.29, 118.20, 106.70, 96.23, 82.48, 60.96, 60.79, 41.09, 36.77, 35.54, 30.18, 25.26, 25.04, 18.84, 14.26; HRMS (ESI-TOF) calcd for $\mathrm{C}_{18} \mathrm{H}_{26} \mathrm{NaO}_{6}[\mathrm{M}+\mathrm{Na}]^{+} 361.1622$, found 361.1622 .

以化合物 13 为原料, 参考本研究组先前已报道工 作 ${ }^{[17]}$ 合成化合物 14.

2,2-二甲基-4-羰基-4,5,6,8,9,10-六氢-5,9a-环氧奥 [5,6- $d][1,3]$-二噁英- $6 a(7 H)$-甲酸乙酯 $(14)$ : 白色固体, 产 率 60\%. m.p. 109 $111{ }^{\circ} \mathrm{C} ;{ }^{1} \mathrm{H}$ NMR $\left(300 \mathrm{MHz}, \mathrm{CDCl}_{3}\right)$ $\delta: 4.95(\mathrm{~d}, J=5.9 \mathrm{~Hz}, 1 \mathrm{H}), 4.17 \sim 4.01(\mathrm{~m}, 2 \mathrm{H}), 3.07$ (d, $J=12.5 \mathrm{~Hz}, 1 \mathrm{H}), 2.68(\mathrm{~d}, J=18.5 \mathrm{~Hz}, 1 \mathrm{H}), 2.38 \sim 2.23(\mathrm{~m}$, $2 \mathrm{H}), 2.03 \sim 1.87(\mathrm{~m}, 3 \mathrm{H}), 1.85 \sim 1.66(\mathrm{~m}, 3 \mathrm{H}), 1.63(\mathrm{~s}$, $3 \mathrm{H}), 1.62(\mathrm{~s}, 3 \mathrm{H}), 1.22(\mathrm{t}, J=7.1 \mathrm{~Hz}, 3 \mathrm{H}) ;{ }^{13} \mathrm{C}$ NMR $(75$ $\left.\mathrm{MHz}, \mathrm{CDCl}_{3}\right) \delta: 175.46,163.53,159.43,106.78,106.67$, 94.77, 72.39, 61.80, 61.31, 46.92, 40.69, 37.09, 34.83, 26.15, 24.23, 23.90, 14.16; FTIR (KBr, thin film) $v: 3425$, 2984, 2949, 1723, 1657, 1441, 1238, 1100, 811, 704, 525 $\mathrm{cm}^{-1}$; HRMS (ESI-TOF) calcd for $\mathrm{C}_{17} \mathrm{H}_{23} \mathrm{O}_{6}[\mathrm{M}+\mathrm{H}]^{+}$ 323.1489 , found 323.1489 .

$3.2 .2(5 R, 6 a R, 7 S, 9 a R)-2,2,7$-三甲基-5, $6 a, 7,8,9,10$-六 氢-5,9a-环氧宷 $[5,6-d][1,3]$-二噁英- $4(6 H)$-酮 $(20)$ 的合 成

参照文献[18]合成中间体(1S,2S)-1-烯丙基-2-甲基5-环戊酮基-1-甲酸乙酯(16), 淡黄色油状液体, 产率 97\%. ${ }^{1} \mathrm{H}$ NMR (400 MHz, $\mathrm{CDCl}_{3}$ ) $\delta: 5.66 \sim 5.53(\mathrm{~m}, 1 \mathrm{H})$, $5.12 \sim 5.08(\mathrm{~m}, 1 \mathrm{H}), 5.06(\mathrm{t}, J=1.1 \mathrm{~Hz}, 1 \mathrm{H}), 4.17(\mathrm{dq}, J=$ 10.8, 7.1 Hz, 1H), 4.09 (dq, $J=10.8,7.1 \mathrm{~Hz}, 1 \mathrm{H}), 2.63$ (ddt, $J=14.2,6.3,1.4 \mathrm{~Hz}, 1 \mathrm{H}), 2.57 \sim 2.43(\mathrm{~m}, 2 \mathrm{H}), 2.30$ $(\mathrm{dq}, J=12.0,6.7 \mathrm{~Hz}, 1 \mathrm{H}), 2.17 \sim 1.98(\mathrm{~m}, 2 \mathrm{H}), 1.86 \sim 1.72$ (m, 1H), 1.23 (t, $J=7.1 \mathrm{~Hz}, 3 \mathrm{H}), 1.02$ (d, $J=6.9 \mathrm{~Hz}, 3 \mathrm{H})$; ${ }^{13} \mathrm{C}$ NMR $\left(100 \mathrm{MHz}, \mathrm{CDCl}_{3}\right) \delta: 216.22,170.51,133.33$, 119.49 , 62.94, 61.09, 39.01, 38.65, 35.70, 28.32, 15.45, 14.32; FTIR (KBr, thin film) v: 2968, 1741, 1454, 1227, 1115, 1019, 924, 860, 762, $587 \mathrm{~cm}^{-1}$; HRMS (ESI-TOF) calcd for $\mathrm{C}_{12} \mathrm{H}_{19} \mathrm{O}_{3}[\mathrm{M}+\mathrm{H}]^{+}$211.1329, found 211.1326.
将化合物 16 (1.32 g, $6.26 \mathrm{mmol})$ 溶解于二甲基亚砜 (DMSO) $(20 \mathrm{~mL})$ 中, 加入 $\mathrm{H}_{2} \mathrm{O}(112.7 \mu \mathrm{L}, 6.26 \mathrm{mmol})$ 和 $\mathrm{LiCl}(530.7 \mathrm{mg}, 12.52 \mathrm{mmol})$, 升温至 $160{ }^{\circ} \mathrm{C}$ 反应 $36 \mathrm{~h}$. 等反应液温度降至室温后, 加入水 $(20 \mathrm{~mL})$ 和二氯甲烷 (DCM) $(15 \mathrm{~mL})$ 萃取. 水相继续用 $\mathrm{DCM}(10 \mathrm{~mL} \times 2)$ 萃 取, 合并有机相. 有机相用无水硫酸镁干燥, 过滤, 用 旋转蒸发仪蒸干溶剂, 硅胶柱层析 $[V$ (石油醚) : $V$ (乙酸 乙酯 $)=10: 1]$, 分离得到 $482.8 \mathrm{mg}(2 R, 3 S)$-2-烯丙基-3甲基环戊-1-酮(18): 无色油状液体，收率 $85 \%, d r=10$ : 1. ${ }^{1} \mathrm{H}$ NMR (400 MHz, $\mathrm{CDCl}_{3}$ ) $\delta: 5.74$ (ddt, $J=17.2,10.0$, $7.2 \mathrm{~Hz}, 1 \mathrm{H}), 5.10 \sim 4.97(\mathrm{~m}, 2 \mathrm{H}), 2.36 \sim 2.34(\mathrm{~m}, 2 \mathrm{H})$, $2.14 \sim 2.01(\mathrm{~m}, 2 \mathrm{H}), 1.96 \sim 1.88(\mathrm{~m}, 1 \mathrm{H}), 1.76 \sim 1.70(\mathrm{~m}$, $1 \mathrm{H}), 1.46 \sim 1.35(\mathrm{~m}, 1 \mathrm{H}), 1.14(\mathrm{~d}, J=6.5 \mathrm{~Hz}, 3 \mathrm{H}), 0.90 \sim$ $0.88(\mathrm{~m}, 1 \mathrm{H}) ;{ }^{13} \mathrm{C}$ NMR $\left(100 \mathrm{MHz}, \mathrm{CDCl}_{3}\right) \delta: 220.25$, 135.62, 116.68, 56.12, 38.07, 36.30, 31.99, 29.55, 19.46; FTIR (KBr, thin film) v: 2960, 1735, 1649, 1451, 1167, 1004, $916 \mathrm{~cm}^{-1}$. HRMS (ESI-TOF) calcd for $\mathrm{C}_{9} \mathrm{H}_{15} \mathrm{O}$ $[\mathrm{M}+\mathrm{H}]^{+}$139.1117, found 139.1118 .

以化合物 18 为原料，按照化合物 13 的合成方法合 成了 6 -(((1R,2R,3S)-2-烯丙基-1-羟基-3-甲基-环戊烷基) 甲基)-2,2-二甲基-4H-1,3-二啞英-4-酮(19)：淡黄色油状 液体，产率 $66 \%$, brsm: $88 \%, d r>20 ： 1 .{ }^{1} \mathrm{H}$ NMR (400 $\left.\mathrm{MHz} \mathrm{CDCl}_{3}\right) \delta: 5.85$ (ddt, $\left.J=17.2,10.0,7.2 \mathrm{~Hz}, 1 \mathrm{H}\right)$, $5.28(\mathrm{~s}, 1 \mathrm{H}), 5.09$ (dt, $J=17.2,1.8 \mathrm{~Hz}, 1 \mathrm{H}), 5.00$ (ddd, $J=$ 10.1, 2.2, $1.1 \mathrm{~Hz}, 1 \mathrm{H}), 2.64$ (d, $J=13.9 \mathrm{~Hz}, 1 \mathrm{H}), 2.35$ $2.23(\mathrm{~m}, 2 \mathrm{H}), 2.23 \sim 2.12(\mathrm{~m}, 1 \mathrm{H}), 1.98 \sim 1.78(\mathrm{~m}, 4 \mathrm{H})$, $1.68(\mathrm{~s}, 6 \mathrm{H}), 1.66 \sim 1.59(\mathrm{~m}, 1 \mathrm{H}), 1.26 \sim 1.08(\mathrm{~m}, 2 \mathrm{H})$, $0.99(\mathrm{~d}, J=6.4 \mathrm{~Hz}, 3 \mathrm{H}) ;{ }^{13} \mathrm{C} \mathrm{NMR}\left(100 \mathrm{MHz}, \mathrm{CDCl}_{3}\right) \delta$ : 169.32 , 161.07, 138.44, 116.01, 106.70, 95.84, 82.23, $55.95,44.86,38.34,38.04,32.87,30.93,25.33,25.27$, 20.41; FTIR (KBr, thin film) v: 2953, 2356, 1720, 1628, 1386, 1275, 1009, 906, 815, 641, $564 \mathrm{~cm}^{-1}$; HRMS (ESITOF) calcd for $\mathrm{C}_{16} \mathrm{H}_{25} \mathrm{O}_{4}[\mathrm{M}+\mathrm{H}]^{+}$281.1747, found 281.1751 .

将烯烃化合物 19 (1.0 g, $3.57 \mathrm{mmol})$ 溶解于 DCM $(10 \mathrm{~mL})$ 中, 在 $-78{ }^{\circ} \mathrm{C}$ 条件下, 往溶液中通入臭氧, 直 到溶液变为蓝色为止. 往溶液中加入 $\mathrm{Et}_{3} \mathrm{~N}(992.5 \mu \mathrm{L}$, $7.14 \mathrm{mmol})$, 在室温条件下搅拌 $12 \mathrm{~h}$. 加入水 $(10 \mathrm{~mL})$ 和 $\mathrm{DCM}(10 \mathrm{~mL})$ 萃取. 水相继续用 $\mathrm{DCM}(10 \mathrm{~mL} \times 2)$ 萃取, 合并有机相. 有机相用饱和食盐水 $(10 \mathrm{~mL})$ 洗涤，无水硫 酸镁干燥，过滤，用旋转蒸发仪蒸干溶剂. 将所得到的 粗产物溶解于 $\mathrm{DCM}(10 \mathrm{~mL})$ 中, $0{ }^{\circ} \mathrm{C}$ 条件下, 加入 $\mathrm{BF}_{3} \cdot \mathrm{OEt}_{2}(906.8 \mu \mathrm{L}, 7.14 \mathrm{mmol})$, 撤去冰浴, 反应液在室 温下继续搅拌 $3 \mathrm{~h}$. 加入饱和 $\mathrm{NaHCO}_{3}(5 \mathrm{~mL})$ 淬灭反应, 加入水 $(10 \mathrm{~mL})$ 和 $\mathrm{DCM}(10 \mathrm{~mL})$ 萃取. 水相继续用 $\mathrm{DCM}$ 
$(10 \mathrm{~mL} \times 2)$ 萃取, 合并有机相. 有机相用无水硫酸镁干 燥, 过滤, 用旋转蒸发仪蒸干溶剂, 硅胶柱层析 $[V($ 石油 醚) $: V$ (乙酸乙酯 $)=10: 1]$, 分离得到 $678.8 \mathrm{mg}(5 R$, $6 a R, 7 S, 9 a R)-2,2,7$-三甲基-5,6a,7,8,9,10-六氢-5,9a-环氧 宷 $[5,6-d][1,3]$-二啞英-4(6H)-酮(20)(两步收率 72\%). 20: 无色油状液体，产率 $72 \%, d r=10: 1 .{ }^{1} \mathrm{H}$ NMR (400 $\left.\mathrm{MHz}, \mathrm{CDCl}_{3}\right) \delta: 4.96(\mathrm{~d}, J=5.6 \mathrm{~Hz}, 1 \mathrm{H}), 2.72(\mathrm{~d}, J=$ $18.0 \mathrm{~Hz}, 1 \mathrm{H}), 2.30$ (dd, $J=11.9,8.4 \mathrm{~Hz}, 1 \mathrm{H}), 2.06$ (d, $J=18.0 \mathrm{~Hz}, 1 \mathrm{H}), 2.01 \sim 1.96(\mathrm{~m}, 1 \mathrm{H}), 1.93 \sim 1.87(\mathrm{~m}$, $1 \mathrm{H}), 1.87 \sim 1.81(\mathrm{~m}, 1 \mathrm{H}), 1.81 \sim 1.67(\mathrm{~m}, 3 \mathrm{H}), 1.61(\mathrm{~d}$, $J=1.7 \mathrm{~Hz}, 6 \mathrm{H}), 1.40 \sim 1.32(\mathrm{~m}, 1 \mathrm{H}), 0.92(\mathrm{~d}, J=6.9$ $\mathrm{Hz}, 3 \mathrm{H}) ;{ }^{13} \mathrm{C} \mathrm{NMR}\left(100 \mathrm{MHz}, \mathrm{CDCl}_{3}\right) \delta: 163.54$, $159.51,107.59,106.66,91.40,73.69,55.66,43.41$, 41.85 , 39.69, 35.26, 33.83, 26.64, 23.60, 20.28; FTIR $(\mathrm{KBr}$, thin film) $v: 3518,2953,2868,1726,1645,1420$, 1269, 1203, 1035, 898, 702, $584 \mathrm{~cm}^{-1}$; HRMS (ESI-TOF) calcd for $\mathrm{C}_{15} \mathrm{H}_{21} \mathrm{O}_{4}[\mathrm{M}+\mathrm{H}]^{+}$265.1435, found 265.1436 .

\section{辅助材料 (Supporting Information) 化合物 14} (CCDC 1895118) 的单晶数据以及化合物 13 14、16、 18 20 的 ${ }^{1} \mathrm{H}$ NMR 和 ${ }^{13} \mathrm{C}$ NMR 图谱. 这些材料可以 免费从本刊网站(http://sioc-journal.cn/)上下载.

\section{References}

[1] (a) Wang, J.; Chen, S. G.; Sun, B. F.; Lin, G. Q.; Shang, Y. J. Chemistry (Weinheim an der Bergstrasse, Germany) 2013, 19, 2539.

(b) Wu, Y. B.; Ni, Z. Y.; Shi, Q. W.; Dong, M.; Kiyota, H.; Gu, Y. C.; Cong, B. Chem. Rev. 2012, 112, 5967.

(c) Zhang, H.; Liu, H. B.; Yue, J. M. Chem. Rev. 2014, 114, 883.

(d) Li, X. X.; Song, W. Z.; Ke, X. N.; Xu, X. F.; Liu, P.; Houk, K. N.; Zhao, X. L.; Tang, W. P. Chem.-Eur. J. 2016, 22, 7079.

(e) Simeonov, S. P.; Nunes, J. P.; Guerra, K.; Kurteva, V. B.; Afonso, C. A. Chem. Rev. 2016, 116, 5744.
[2] (a) Akee, R. K.; Ransom, T.; Ratnayake, R.; McMahon, J. B.; Beutler, J. A. J. Nat. Prod. 2012, 75, 459.

(b) Ratnayake, R.; Covell, D.; Ransom, T. T.; Gustafson, K. R.; Beutler, J. A. Org. Lett. 2009, 11, 57.

[3] (a) Miski, M.; Deluengo, D. H.; Mabry, T. J. Phytochemistry 1987, 26, 199.

(b) Blanco, J. G.; Gil, R. R.; Alvarez, C. I.; Patrito, L. C.; GentiRaimondi, S.; Flury, A. FEBS Lett. 1997, 409, 396.

[4] (a) Hikino, H.; Ito, K.; Takemoto, T. Chem. Pharm. Bull. 1968, 16, 1608 .

(b) Yang, Y.; Kinoshita, K.; Koyama, K.; Takahashi, K.; Tai, T.; Nunoura, Y.; Watanabe, K. Phytomedicine 1999, 6, 89.

[5] (a) Rosselli, S.; Maggio, A.; Bellone, G.; Bruno, M. Tetrahedron Lett. 2006, 47, 7047.

(b) Rasmussen, U.; Brøogger Christensen, S.; Sandberg, F. Acta Pharm. Suec. 1978, 15, 133.

(c) Huang, S. Z.; Zhang, X.; Ma, Q. Y.; Zheng, Y. T.; Dai, H. F.; Wang, Q.; Zhou, J.; Zhao, Y. X. RSC Adv. 2015, 5, 80254.

[6] Navickas, V.; Ushakov, D. B.; Maier, M. E.; Strobele, M.; Meyer, H. J. Org. Lett. 2010, 12, 3418

[7] Hirose, T.; Miyakoshi, N.; Mukai, C. J. Org. Chem. 2008, 73, 1061.

[8] Jouanneau, M.; Bonepally, K. R.; Jeuken, A.; Tap, A.; Guillot, R.; Ardisson, J.; Ferezou, J. P.; Prunet, J. Org. Lett. 2018, 20, 2176.

[9] Li, W.; Liu, X. Z.; Zhou, X. F.; Lee, C. S. Org. Lett. 2010, 12, 548.

[10] Mascarenas, J. L.; Varela, I.; Lopez, F. Acc. Chem. Res. 2019, 52, 465.

[11] Parmar, D.; Matsubara, H.; Price, K.; Spain, M.; Procter, D. J. J. Am. Chem. Soc. 2012, 134, 12751.

[12] Nicolaou, K. C.; Kang, Q. A.; Ng, S. Y.; Chen, D. Y. K. J. Am. Chem. Soc. 2010, 132, 8219.

[13] Bao, W. L.; Tao, Y. Z.; Cheng, J. Q.; Huang, J. R.; Cao, J. M.; Zhang, M. X.; Ye, W. J.; Wang, B.; Li, Y.; Zhu, L. Z.; Lee, C. S. Org. Lett. 2018, 20, 7912.

[14] Li, G. J.; Xu, X. L.; Tian, H. C.; Liu, X. T.; Chen, W.; Yang, X. D.; Zhang, H. B. RSC Adv. 2017, 7, 50822.

[15] Han, Z. B.; Wang, Z.; Ding, K. L. Adv. Synth. Catal. 2011, 353, 1584.

[16] Chen, W.; Yang, X. D.; Tan, W. Y.; Zhang, X. Y.; Liao, X. L.; Zhang, H. Angew. Chem., Int. Ed. 2017, 56, 12327.

[17] Wang, M. S.; Wang, Z.; Chen, W.; Yang, X. D.; Zhang, H. B. Org. Lett. 2019, 21, 1881.

[18] Shen, Y.; Li, L.; Pan, Z.; Wang, Y.; Li, J.; Wang, K.; Wang, X.; Zhang, Y.; Hu, T.; Zhang, Y. Org. Lett. 2015, 17, 5480.

[19] (a) Krapcho, A. P. Synthesis-Stuttgart 1982, 805. (b) Krapcho, A. P. Synthesis-Stuttgart 1982, 893. 